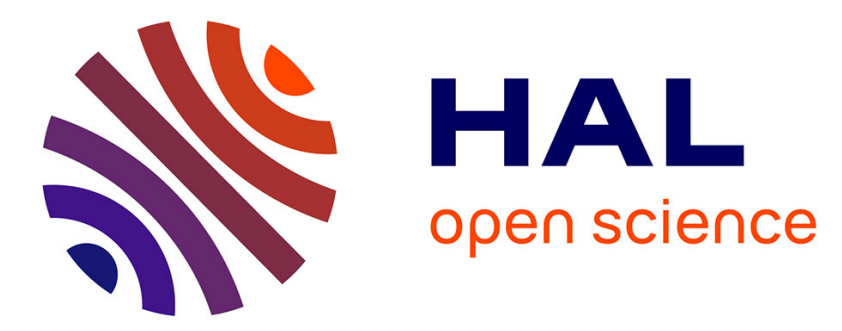

\title{
Description par l'opérateur densité du mouvement statistique de l'exciton électronique dans une assemblée d'agrégats moléculaires orientés dans une matrice non résonnante \\ C. Aslangul, P. Kottis
}

\section{To cite this version:}

C. Aslangul, P. Kottis. Description par l'opérateur densité du mouvement statistique de l'exciton électronique dans une assemblée d'agrégats moléculaires orientés dans une matrice non résonnante. Journal de Physique Lettres, 1977, 38 (20), pp.409-412. 10.1051/jphyslet:019770038020040900 . jpa00231408

\section{HAL Id: jpa-00231408 \\ https://hal.science/jpa-00231408}

Submitted on 1 Jan 1977

HAL is a multi-disciplinary open access archive for the deposit and dissemination of scientific research documents, whether they are published or not. The documents may come from teaching and research institutions in France or abroad, or from public or private research centers.
L'archive ouverte pluridisciplinaire HAL, est destinée au dépôt et à la diffusion de documents scientifiques de niveau recherche, publiés ou non, émanant des établissements d'enseignement et de recherche français ou étrangers, des laboratoires publics ou privés. 


\title{
DESCRIPTION PAR L'OPÉRATEUR DENSITÉ DU MOUVEMENT STATISTIQUE DE L'EXCITON ÉLECTRONIQUE DANS UNE ASSEMBLÉE D'AGRÉGATS MOLÉCULAIRES ORIENTÉS DANS UNE MATRICE NON RÉSONNANTE
}

\author{
C. ASLANGUL \\ Centre de Mécanique Ondulatoire Appliquée, 23, rue du Maroc, 75019 Paris, France \\ et \\ P. KOTTIS \\ Laboratoire d'Optique Moléculaire (E.R. 134), Université de Bordeaux I, \\ 351, cours de la Libération, 33405 Talence Cedex, France
}

(Reçu le 8 juillet 1977, révisé le 15 septembre 1977, accepté le 19 septembre 1977)

\begin{abstract}
Résumé. - Le mouvement excitonique, dont la description a été définie dans des publications antérieures [1, 2], est ici généralisé au cas d'un agrégat contenant un nombre quelconque de sites, $N$.

Abstract. - We give a generalization of the excitonic motion, as previously defined [1, 2], for a molecular aggregate containing an arbitrary number of sites, $N$.
\end{abstract}

1. Introduction. - Nous proposons ici une extension de l'étude entreprise précédemment [1], concernant le mouvement de l'exciton dans un agrégat moléculaire fini inséré dans une matrice isotopique non résonnante, support d'un champ de phonons susceptibles d'interagir avec les excitations électroniques élémentaires de l'agrégat.

Le problème est le suivant : dans l'hypothèse où l'on sait (à l'aide d'un piège énergétique, ou par interférence d'excitations optiques ou par excitation avec des particules chargées) créer un état initial où toute l'excitation est concentrée sur un seul site, quelle est l'évolution ultérieure de cette quasi-particule d'énergie? Une analyse intuitive affirme que, en raison des couplages résiduels entre sites, l'excitation se déplace d'une molécule à l'autre à une fréquence donnée en gros par l'énergie/ $h$ d'interaction entre sites. Nous considérons cette approche comme incorrecte pour au moins deux raisons : d'une part, elle ignore la notion de cohérence électronique, d'autre part, elle fait fi des corrélations quantiques existant entre deux sous-systèmes d'un même système quantique; on peut donc la qualifier de classique et d'ailleurs ses conclusions sont fausses. Si l'on décrit convenablement le système en Mécanique Quantique, on obtient un mouvement extrêmement complexe qui est celui d'un paquet d'ondes dans un milieu dispersif. Notamment, la taille de la particule est en général grande devant la distance entre molécules de sorte que l'expression transfert de site à site ne doit pas être prise à la lettre. Dans le cas d'un dimère [1], les résultats quantiques permettent de réhabiliter d'une certaine façon l'image classique du transfert : on montre ici que cette situation est très particulière et ne se rencontre plus dès que l'on considère un trimère.

Rappelons que la description de ces phénomènes complexes et mal compris, comme par exemple la fusion entre excitons, utilise des modèles admettant comme hypothèses les conclusions naïves évoquées ci-dessus, que nous démontrons incorrectes: de ce fait, la discussion précise du transfert microscopique présente un intérêt évident.

Le formalisme utilisé repose sur l'opérateur densité : ceci est inévitable si l'on veut introduire d'une façon ou d'une autre la composante du mouvement résultant de l'interaction exciton-phonon.

Le caractère spécifique du système étudié est sa dimension finie : de ce fait, on ne peut utiliser des conditions aux limites cycliques et il n'apparait pas de grandeur jouant le rôle d'une quasi-impulsion résultant de la symétrie de translation. Ces deux aspects du problème ont fait la complexité et ont justifié largement le recours au langage des superopérateurs, car on ne rencontre alors que des déductions utilisant des techniques algébriques extrêmement banales 
ce formalisme a été complètement précisé précédemment $[1,2]$.

Nous poursuivons ici l'étude entreprise dans des publications précédentes [1, 2], en la généralisant à un agrégat contenant un nombre quelconque, $N$, de sites. Le modèle utilisé est celui de Haken et Strobl [3], avec abandon délibéré des conditions cycliques de Born-Von Karman, puisque nous traitons spécifiquement le cas de petits systèmes.

Le formalisme est celui des superopérateurs, déjà décrit $[1,4]$ : la sous-algèbre contenant toute l'information qui concerne le transfert d'énergie dans l'état excité, soit $\mathrm{A}^{*}[5]$, est engendrée par les $N^{2}$ vecteurs $\left.\left.\left.\mid Y_{p q}\right) \equiv|| M_{p}\right\rangle\left\langle M_{q}\right|\right), 1 \leqslant p, q \leqslant N$, où $\left|M_{p}\right\rangle$ est le vecteur monoexcité antisymétrisé construit avec le premier état excité du $p$-ième site. Dans ce sous-espace, la restriction d'évolution (voir équation (3.4) de la référence [1]) s'écrit :

$$
\left.\left.\left.i \hbar \frac{\mathrm{d}}{\mathrm{d} t} \mid \rho^{*}(t)\right)=\left(\hat{H}_{0^{-}}^{*}-i \hbar \hat{\Gamma}^{*}\right) \mid \rho^{*}(t)\right) \equiv \hat{H}^{*} \mid \rho^{*}(t)\right)
$$

Les opérateurs $\hat{H}_{0^{-}}$et $\hat{\Gamma}$ ont déjà été définis [1] et représentent respectivement le couplage quantique entre sites (réduit aux plus proches voisins) et la relaxation induite par l'interaction entre l'exciton et les vibrations site-site et site-réseau : les opérateurs avec astérisque sont les restrictions à $A^{*}$.

$\mathrm{Si}$ on désigne par $\hbar \Delta \omega$ le couplage entre deux voisins, $\hat{H}_{0}$ s'écrit :

$$
\begin{aligned}
\hat{H}_{0}= & \hbar \omega_{0} \sum_{p=1}^{N}\left|M_{p}\right\rangle\left\langle M_{p}\right|+ \\
& +\hbar \Delta \omega \sum_{p=1}^{N-1}\left|M_{p}\right\rangle\left\langle M_{p+1}|+| M_{p+1}\right\rangle\left\langle M_{p}\right|
\end{aligned}
$$

et ceci entraîne :

$$
\left.\hat{H}_{0^{-}}^{*}=\hbar \Delta \omega \sum_{p, q} \sum_{p^{\prime}, q^{\prime}} \mid Y_{p q}\right)\left\{\left(\delta_{p+1 p^{\prime}}+\delta_{p-1 p^{\prime}}\right) \delta_{q q^{\prime}}-\left(\delta_{q+1 q^{\prime}}+\delta_{q-1 q^{\prime}}\right) \delta_{p p^{\prime}}\right\}\left(Y_{p^{\prime} q^{\prime}} \mid\right.
$$

Par ailleurs, avec les hypothèses habituelles du modèle stochastique [3], il est facile d'établir l'expression suivante pour l'opérateur de relaxation :

$$
\begin{aligned}
& \left.\hat{\Gamma}^{*}=\sum_{p, q} \mid Y_{p q}\right)\left\{2\left(1-\delta_{p q}\right) \gamma_{0}+\left(4-\delta_{p 1}-\delta_{p N}-\delta_{q 1}-\delta_{q N}\right) \gamma_{1}\right\}\left(Y_{p q} \mid+\right. \\
& \left.\quad+2 \gamma_{1} \sum_{p, q} \sum_{p^{\prime}, q^{\prime}} \mid Y_{p q}\right)\left\{\left(\delta_{p p^{\prime}-1}+\delta_{p p^{\prime}+1}\right) \delta_{p q} \delta_{p^{\prime} q^{\prime}}+\delta_{p p^{\prime}-1} \delta_{q q^{\prime}+1} \delta_{p q-1}+\delta_{p p^{\prime}+1} \delta_{q q^{\prime}-1} \delta_{p q+1}\right\}\left(Y_{p^{\prime} q^{\prime}} \mid .\right.
\end{aligned}
$$

Les symboles de Kronecker apparaissant dans la première ligne proviennent du fait que la chaîne est de longueur finie, et sont donc des effets de bords.

On a montré que, si le recouvrement spatial entre sites est faible et si $|\Delta \omega| \ll \omega_{0}$, alors les moments calculés avec la densité d'énergie $E\left(\mathbf{r}, \mathbf{r}^{\prime} ; t\right)$ ne dépendent explicitement que des éléments diagonaux du type $\left(Y_{p p} \mid \rho^{*}(t) \equiv \rho_{p p}(t)\right.$; il faut toutefois noter qu'ils dépendent implicitement de tous les éléments de matrice de l'opérateur densité par l'apparition du couplage quantique entre sites dans l'équation d'évolution. En tout état de cause, il est utile de définir une fonction génératrice des moments, $\rho_{0}(\varphi ; t)$ :

$$
\rho_{0}(\varphi ; t)=\sum_{p=1}^{N} \exp \left[i\left(p-\frac{N+1}{2}\right) \varphi X\right] \rho_{p p}(t)
$$

où $X$ est la distance entre deux sites adjacents ; le $n$-ième moment s'exprime alors par :

$$
M_{n}=i^{-n}\left\{\frac{\partial^{n}}{\partial \varphi^{n}} \rho_{0}(\varphi ; t)\right\}_{\varphi=0}
$$

Dans cette note, on se borne à donner les résultats correspondant aux cas extrêmes des mouvements purement cohérent et purement incohérent; le mouvement général sera détaillé dans une autre publication. On admet que l'état initial est parfaitement localisé, $q$ étant l'indice du site porteur de l'excitation à cet instant : on sait que, s'agissant d'une préparation optique, ceci n'a de sens que si le système champ + agrégat possède des propriétés très particulières [8] (champ à grande dispersion spectrale et polarisation adaptée au défaut orientationnel de l'agrégat).

2. Mouvement purement cohérent. - Dans ce cas, l'interaction exciton phonon est négligeable, ce qui revient à annuler l'opérateur de relaxation $\hat{\Gamma}$. On trouve alors immédiatement :

$\rho_{0}(\varphi: t)=\left(\frac{2}{N+1}\right)^{2} \sum_{l, m=1}^{N} \sum_{p=1}^{N} \exp \left[i\left(p-\frac{N+1}{2}\right) \varphi X\right] \sin l p \theta \sin m p \theta \sin l q \theta \sin m q \theta \times$

$$
\times \mathrm{e}^{2 i \Delta \omega(\cos 1 \theta-\cos m \theta) t}\left(\theta=\frac{\pi}{N+1}\right)
$$


Une première différence, par rapport aux résultats du dimère [1] apparaît lorsque l'on examine les récurrences du mouvement : ici, la fonction génératrice est une combinaison linéaire de fonctions harmoniques de fréquences non commensurables : sauf dans le cas du trimère, il n'existe pas de période stricte finie : on peut cependant définir une quasi-période, $T^{*}$, par les conditions :

$$
M_{1}\left(T^{*}\right) \simeq\left(q-\frac{(N+1)}{2}\right) X \Delta\left(T^{*}\right) \equiv\left(M_{2}-M_{1}^{2}\right)^{1 / 2} \simeq 0 .
$$

La figure 1 montre la variation du logarithme de $T^{*}$ en fonction du nombre $N$ de sites, lorsque l'excitation se trouve initialement à l'une des extrémités de l'agrégat $(q=1)$. On observe une augmentation brutale au voisinage de $N=10$ : pour un état

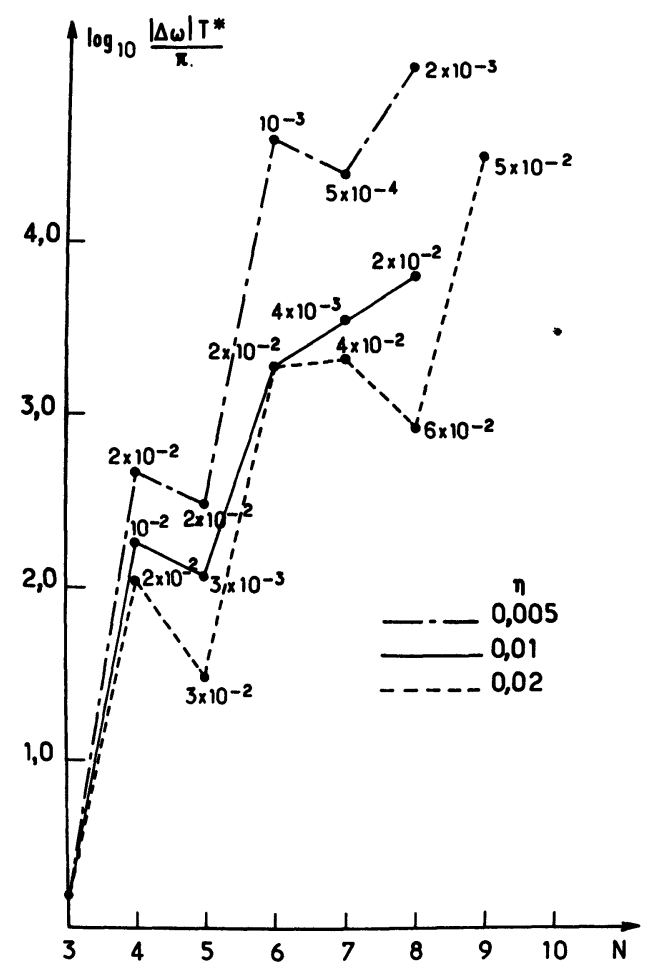

FIG. 1. - Variation du logarithme de la quasi-période $T$ en fonction du nombre de sites $N$. Au voisinage de chaque point est est indiquée la valeur de l'entropie de délocalisation égale à $\sum_{p} \rho_{p p}(t) \log \rho_{p p}(t) ; \eta$ est la mesure de la quasi-récurrence d'autant plus grand que celle-ci est approchée.

[Logarithmic variation of pseudoperiod $T$ versus the number of sites $N$. We indicate, in the vicinity of each point, the value of the delocalization entropy given by $\sum_{p} \rho_{p p}(t) \ln \rho_{p p}(t) ; \eta$ indicates the measure of the pseudorecurrence.] à durée de vie ordinaire, la quasi-récurrence n'a de fait pas le temps de se produire. Une étude détaillée montre par ailleurs une dépendance de $T^{*}$ par rapport à $q$.

Une deuxième différence importante est l'absence de contraction excitonique en général lorsque le centre du paquet d'ondes se trouve sur un site: on observe au plus un tel phénomène, et encore pas toujours, lorsque l'exciton se trouve à l'une des limites de la chaîne : la contraction sur chaque site obtenue pour le dimère $[1,2]$ est donc spécifique de ce système.

La comparaison avec les travaux antérieurs ne peut se faire qu'au voisinage de $t=0$, puisqu'ici tous les moments sont bornés (ils tendent vers $+\infty$ pour une chaîne infinie $[6,7])$. On trouve :

$$
\Delta^{2}(t \simeq 0) \simeq\left(2-\delta_{q 1}-\delta_{q N}\right) \Delta \omega^{2} t^{2} X^{2}
$$

et ce résultat montre que la variation initiale de la dispersion est ici fonction du site porteur de l'excitation initiale.

D'une façon générale, le mouvement excitonique est très complexe (voir la figure 2), notamment en raison de la possibilité de reflections en bout de chaîne: dans la phase intermédiaire, on constate que l'excitation est le plus souvent délocalisée sur une partie importante de l'espace; se représenter le mouvement comme celui d'une particule sautant de site en site n'est donc pas justifié.

3. Mouvement purement incohérent. - Dans ce cas, on suppose la température tellement élevée que l'on peut négliger tous les couplages statiques devant leurs fluctuations quadratiques moyennes : $\hat{H}_{0}$ - est alors identiquement nul de sorte que la dimension matricielle de l'équation d'évolution se réduit à $N$. Un calcul simple conduit alors au résultat :

$$
\begin{aligned}
\rho_{0}(\varphi: t)=\frac{\sin N \frac{\varphi}{2} X}{N \sin \frac{\varphi}{2} X}-2 \frac{i}{N} \sin \frac{\varphi}{2} X \sum_{j=1}^{N} \frac{\exp \left(-i N \frac{\varphi}{2} X\right)-(-1)^{j} \exp \left(+i N \frac{\varphi}{2} X\right)}{\cos \varphi X-\cos j \theta^{\prime}} \times \\
\quad \times \cos \frac{j}{2} \theta^{\prime} \cos j\left(q-\frac{1}{2}\right) \theta^{\prime} \exp \left[-8 \gamma_{1} t\left(\sin \frac{j}{2} \theta^{\prime}\right)^{2}\right]\left(\theta^{\prime}=\frac{\pi}{N}\right) .
\end{aligned}
$$



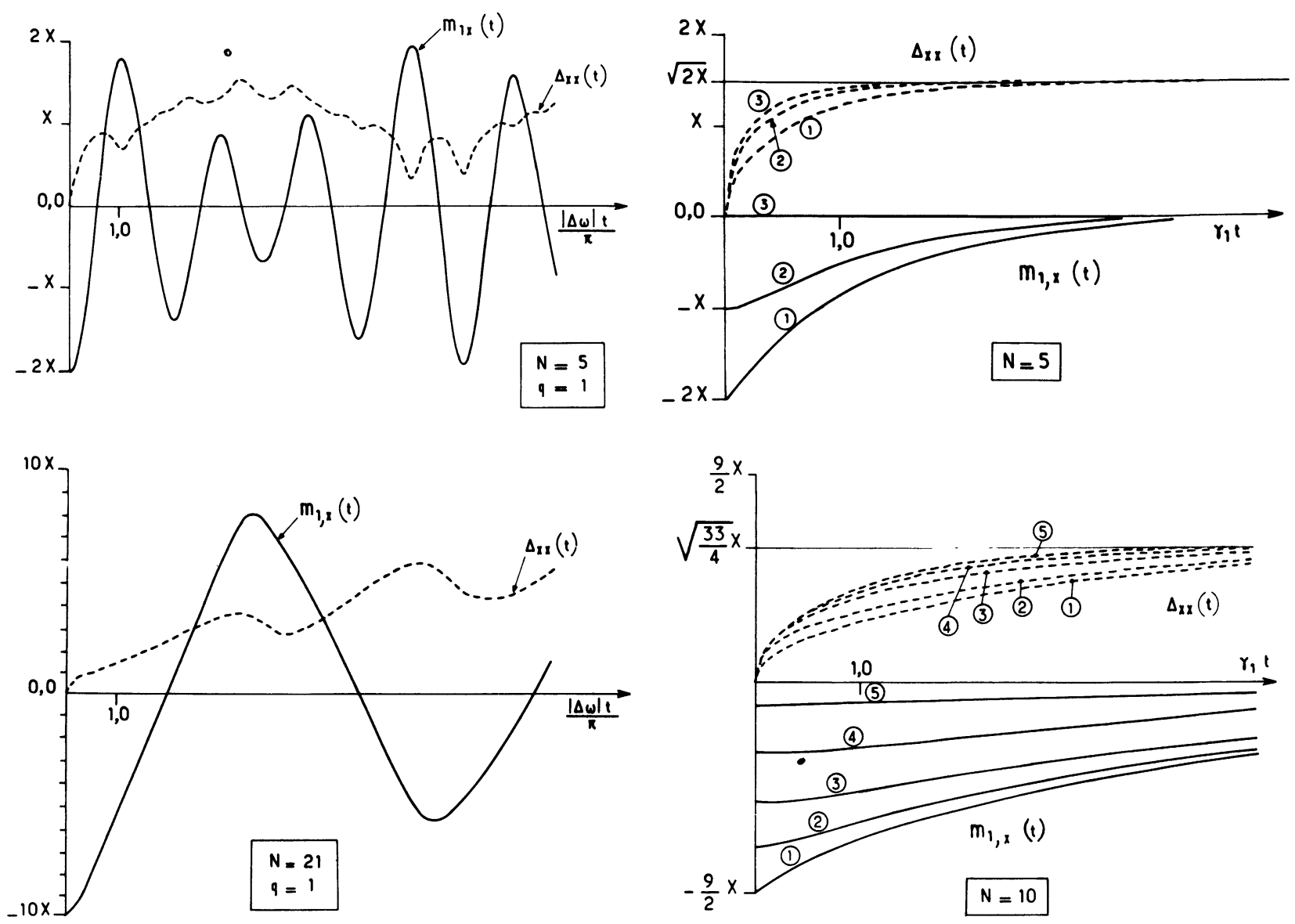

Fig. 2. - Variation du premier moment et de la dispersion dans le cas purement cohérent pour deux chaînes de longueur différente : $N=5$ sites et $N=21$ sites.

[Variation of the first moment and of the dispersion, in the case of a purely coherent motion, for two chains of different length : $N=5$ sites and $N=21$ sites.]

On retrouve ici le même comportement que pour le dimère (voir Fig. 3) : $M_{1}(t)$ et $\Delta(t)$ tendent uniformément vers leurs valeurs d'équilibre, caractéristiques d'une délocalisation complète. Au voisinage de $t=0$, on a :

$$
\Delta^{2}(t \simeq 0) \simeq 2 \gamma_{1} X^{2}\left(2-\delta_{q 1}-\delta_{q N}\right) t
$$

de sorte que le mouvement diffusionnel est défini par la constante $D$ dépendant explicitement de l'endroit où se trouve l'excitation initialement :

$$
D=2 \gamma_{1} X^{2}\left(2-\delta_{q 1}-\delta_{q N}\right)
$$

FIG. 3. - Variation du premier moment et de la dispersion dans le cas du mouvement purement incohérent pour deux chaînes de longueur différente : $N=5$ sites et $N=10$ sites (chaque courbe est indiquée par le numéro du site porteur de l'excitation à l'instant initial).

[Variation of the first moment and of the dispersion, in the case of purely incoherent motion, for two chains of different lenght : $N=5$ sites and $N=10$ sites (the number by each curve indicates the site which carries the excitation at the initial instant).]

Dans ce cas comme dans l'autre, il n'est pas licite de visualiser le mouvement par analogie avec celui d'une particule très petite devant la distance entre deux sites voisins sautant aléatoirement de l'un à l'autre.

Le traitement du cas général et le détail du traitement des deux situations esquissées ci-dessus feront l'objet d'une publication ultérieure.

\section{Bibliographie}

[1] Aslangul, Cl., Kottis, Ph., Phys. Rev. B 10 (1974) 4364.

[2] Aslangul, Cl., Kottis, Ph., C. R. Hebd. Séan. Acad. Sci. B 278 (1974) 735.

[3] Haken, H., Strobl, G., dans The Triplet State, édité par A. B. Zahlan (Cambridge University Press) 1968.

[4] Aslangul, Cl., Kotris, Ph., C. R. Hebd. Séan. Acad. Sci. B 278 (1974) 33.
[5] Aslangul, Cl., Kottis, Ph., Phys. Rev. B 13 (1976) 5544.

[6] Grover, M. K., Silbey, R., J. Chem. Phys. 54 (1971) 4843.

[7] Reineker, P., Phys. Lett. 42A (1973) 389; ibid. 44A (1973) 429.

[8] Aslangul, Cl., Thèse d'Etat, Paris VI, avril 1977. 